PROCEEDINGS OF THE

AMERICAN MATHEMATICAL SOCIETY

Volume 133, Number 5, Pages 1497-1504

S 0002-9939(04)07706-8

Article electronically published on November 1, 2004

\title{
POSITIVE SCALAR CURVATURE AND MINIMAL HYPERSURFACES
}

\author{
HARISH SESHADRI
}

(Communicated by Richard A. Wentworth)

\begin{abstract}
We show that the minimal hypersurface method of Schoen and Yau can be used for the "quantitative" study of positive scalar curvature. More precisely, we show that if a manifold admits a metric $g$ with $s_{g} \geq|T|$ or $s_{g} \geq|W|$, where $s_{g}$ is the scalar curvature of $g, T$ any 2-tensor on $M$ and $W$ the Weyl tensor of $g$, then any closed orientable stable minimal (totally geodesic in the second case) hypersurface also admits a metric with the corresponding positivity of scalar curvature. A corollary pertaining to the topology of such hypersurfaces is proved in a special situation.
\end{abstract}

\section{INTRODUCTION}

In the study of the topology and geometry of Riemannian manifolds with positive scalar curvature, two approaches have played a fundamental role. The first one was initiated by Lichnerowicz in 1961 [6] and is based on the Bochner-Weitzenbock formula for the Dirac operator acting on spinors. This method was greatly developed in a series of papers by Gromov and Lawson [3]. The second method made its appearance in the paper of Schoen and Yau in 1979 [7] and used the theory of minimal hypersurfaces. One of the applications of these methods was to the question of which manifolds admit complete metrics of positive scalar curvature.

It is natural to seek "quantitative" versions of the above "qualitative" question. Our notion of "quantitative" is the following: let $(M, g)$ be a Riemannian manifold and $s$ (or $s_{g}$, if we want to emphasize the dependence on $g$ ) the scalar curvature of $g$. If $f: M \rightarrow \mathbb{R}$ is a function (depending on $g$ and not necessarily smooth), we are interested in the topological implications of the condition $s \geq f$. In this paper we consider functions $f=f_{g}$ that are of conformal weight -2 in the terminology of [5]. This means that $f$ is a function that depends on the metric $g$ in the following way: $f_{u^{2} g}=u^{-2} f_{g}$.

It can be shown (cf. 5]) that, if $f$ is Lipschitz continuous, the conformal class of every metric contains a $C^{2, \alpha}$ metric for which $s>f, s<f$ or $s=f$ and these possibilities are mutually exclusive. Hence the condition " $s \geq f$ " is actually a conformal notion.

Received by the editors August 27, 2003 and, in revised form, November 19, 2003 and January 16, 2004.

2000 Mathematics Subject Classification. Primary 53C21.

(C)2004 American Mathematical Society Reverts to public domain 28 years from publication 1497 
We consider three examples of such $f$ :

1) $f=|T|_{g}$ where $T$ is a 2-tensor on $M$ and $|T|_{g}$ denotes the pointwise norm of $T$ with respect to $g$.

2) $f=\left|W_{g}\right|_{g}$ where $W_{g}$ is the Weyl tensor of $g$ and $|W|_{g}$ again denotes pointwise norm.

3) Let $i: \Sigma^{n-1} \rightarrow M^{n}$ be a hypersurface, with $i$ being the inclusion map. Fix a Riemannian metric $h$ on $\Sigma$, and let $g$ be a fixed extension of $h$ to $M$. Let $u$ be any smooth positive function on $\Sigma$, and let $v$ denote any extension of $u$ to a neighbourhood of $\Sigma$. Denote the second fundamental form and mean curvature (with respect to a fixed normal vector field) of $\Sigma$ in $(M, g)$ by $B_{g}$ and $H_{g}$ respectively. If $L_{u^{2} h}=\left(B_{v^{2} g}-\frac{H_{v^{2} g}}{n-1} u^{2} h\right)$ denotes the trace-free part of the second fundamental form of $\left(\Sigma, u^{2} h\right)$ in $\left(M, v^{2} g\right)$, then it can be checked that $L$ does not depend on the extension $v$. Also,

$$
\left|L_{u^{2} h}\right|_{u^{2} h}^{2}=u^{-2}\left|L_{h}\right|_{h}^{2}
$$

Hence, with $h$ and $g$ as above, the function $f=\left|L_{h}\right|_{h}^{2}$ is of conformal weight -2 .

When $T$ is a closed 2-form on $M$, Gursky and LeBrun [5] used a variation of the spinor method to obtain obstructions to the existence of metrics with $s \geq|T|$ and applied this for the calculation of the Yamabe invariant of certain 4-manifolds. The purpose of this note is to show that the minimal hypersurface method also yields information in the context of quantitative positive scalar curvature and to derive some geometric corollaries. More precisely, we have

Theorem 1.1. Let $(M, g)$ be an orientable Riemannian $n$-manifold with $n \geq 3$. Assume that $s_{g} \geq|T|_{g}$ where $T$ is a smooth 2 -tensor on $M$. If $i: \Sigma \rightarrow M$ is a closed, orientable, stable minimal hypersurface in $M$, then one of the two possibilities holds (where $L_{h}$ is interpreted as in Example 3)):

(i) $\Sigma$ admits a $C^{2, \alpha}$ metric $h$ conformal to $i^{*}(g)$ with $s_{h}>\left|i^{*}(T)\right|_{h}+\left|L_{h}\right|_{h}^{2}$.

(ii) The induced metric $h$ on $\Sigma$ satisfies $s_{h} \equiv\left|i^{*}(T)\right|_{h}+\left|B_{h}\right|_{h}^{2}$.

A similar result holds for $f=|W|$ :

Theorem 1.2. Let $(M, g)$ be an orientable Riemannian $n$-manifold with $n \geq 5$. Assume that $s_{g} \geq c\left|W_{g}\right|_{g}$, where $c \in \mathbb{R}^{+}$. If $i: \Sigma \rightarrow M$ is a closed, orientable, stable totally geodesic hypersurface in $M$, then one of the two possibilities holds:

(i) $\Sigma$ admits a $C^{2, \alpha}$ metric $h$ conformal to $i^{*}(g)$ with $s_{h}>c\left|W_{h}\right|_{h}$.

(ii) The induced metric $h$ on $\Sigma$ satisfies $s_{h} \equiv c\left|W_{h}\right|_{h}$.

In (ii) of both these theorems, the proofs give more information than what has been stated above. We refer the reader to the proofs for details.

Combining Theorem 1.2 with the Böchner formula for harmonic 2-forms on 4manifolds, we obtain the following corollary.

Corollary 1.3. Let $(M, g)$ be an orientable Riemannian 5-manifold with $s \geq$ $2 \sqrt{6}|W|$. If $i: \Sigma \rightarrow M$ is a closed orientable stable totally geodesic hypersurface, then either $H^{2}(\Sigma, \mathbb{R})=0$ or $\left(\Sigma, i^{*}(g)\right)$ is a self-dual Kähler surface of non-negative scalar curvature.

Note that a theorem of Derdzinski [2] states that any compact self-dual Kähler surface is locally symmetric. 
As examples of 5-manifolds $(M, g)$ satisfying the hypotheses of Corollary 1.3 we can consider $(M, g)=\left(\Sigma \times S^{1}, h+d \theta^{2}\right)$, where $(\Sigma, h)$ is (up to scaling) either $S^{4}$ with its standard metric, a closed flat 4 -manifold or $\mathbb{C} P^{2}$ with the Fubini-Study metric. It is clear that the condition $s \geq 2 \sqrt{6}|W|$ is satisfied for the cases of $S^{4}$ and flat 4-manifolds because the Weyl tensor of a Riemannian product of a constant sectional curvature space with $S^{1}$ is zero. The verification for the $\mathbb{C} P^{2}$ case is the remark following the proof of Theorem 1.2 in Section 2. Note that for this case $s \equiv 2 \sqrt{6}|W|=2 \sqrt{6}\left|W^{+}\right| \neq 0$, where $W^{+}$is the self-dual part of the Weyl tensor. It is well known that $s=2 \sqrt{6}\left|W^{+}\right|$for any Kähler surface $(\Sigma, h)$ with non-negative scalar curvature and with the natural orientation; cf. [2. However, according to Corollary [1.3, only the self-dual ones can possibly arise as stable totally geodesic hypersurfaces in 5-manifolds satisfying $s \geq 2 \sqrt{6}|W|$.

For the next corollary we note that if a manifold $M$ has Ric $\geq 0$, then the second variation formula for the area functional (see (2) in Section 2) implies that any stable minimal hypersurface in $M$ is totally geodesic. Combining this observation with the existence of orientable stable minimal hypersurfaces representing codimension-one homology classes in $M$ (when $M$ is orientable and $\operatorname{dim} M \leq 7$ ) and Corollary 1..3 we get

Corollary 1.4. Let $(M, g)$ be an orientable Riemannian 5-manifold with Ric $\geq 0$ and $s \geq 2 \sqrt{6}|W|$. Then every homology class $\alpha$ in $H_{4}(M, \mathbb{R})$ is represented by a hypersurface $\Sigma_{\alpha}$ with $H^{2}\left(\Sigma_{\alpha}, \mathbb{R}\right)=0$ or by a self-dual Kähler surface of nonnegative scalar curvature.

\section{Proofs}

Convention. For the rest of the paper we follow the following convention. For any tensor $S$ on $M$ and any submanifold $i: \Sigma \rightarrow M$,

$$
\text { we denote the tensor } i^{*}(S) \text { on } \Sigma \text { by } \tilde{S} \text {. }
$$

Proof of Theorem [1.1. The proof is based on that of Schoen and Yau in [7].

The object of our study will be the modified scalar curvature $\sigma(g, f)$ corresponding to a function $f_{g}$ of conformal weight -2 defined by

$$
\sigma(g, f)=s_{g}-f_{g} .
$$

We begin by noting that, under a conformal change of the metric $g \rightarrow \bar{g}=u^{\frac{4}{n-2}} g$, the modified scalar curvature transforms by

$$
\sigma(g, f) \rightarrow \sigma(\bar{g}, f)=u^{\frac{-4}{n-2}} \sigma(g, f)-4 \frac{n-1}{n-2} u^{-\frac{n-2}{n+2}} \triangle u .
$$

This follows immediately from the corresponding transformation law for the usual scalar curvature $s$; cf. [1].

The stability of the minimal surface $\Sigma$ implies that for any smooth function $\phi$ on $\Sigma$,

$$
-\int_{\Sigma}\left(\operatorname{Ric}(N, N)+|B|^{2}\right) \phi^{2}+\int_{\Sigma}|\nabla \phi|^{2} \geq 0
$$

where $N$ is a unit normal vector field on $\Sigma$, Ric is the Ricci tensor of $g$ and $B$ is the second fundamental form of $\Sigma$. 
As in [7, the Gauss curvature equations and the minimality of $\Sigma$ can be used to write the above inequality as

$$
\int_{\Sigma} \frac{s \phi^{2}}{2}-\int_{\Sigma} \frac{s_{\tilde{g}} \phi^{2}}{2}+\frac{1}{2} \int_{\Sigma}|B|^{2} \phi^{2} \leq \int_{\Sigma}|\nabla \phi|^{2},
$$

where $s_{\tilde{g}}$ is the scalar curvature of $\Sigma$ with the induced metric.

To adapt (3) for modified scalar curvature, we need the following simple lemma.

Lemma 2.1. Let $S \in \otimes^{r} T^{*} M^{n}$ be a tensor on $M$ and $i: \Sigma^{k} \rightarrow M$ a submanifold, with $i$ being the inclusion map. Then $|\tilde{S}|_{\tilde{g}} \leq|S|_{g}$ at any point of $\Sigma$.

Proof. Let $p \in \Sigma$. Let $\left\{e_{1}, . ., e_{k}\right\}$ be an orthonormal basis, with respect to $\tilde{g}$, of $T_{p} \Sigma$, where $k=\operatorname{dim} \Sigma$. Extend it to an orthonormal basis $\left\{e_{1}, . ., e_{n}\right\}$ of $T_{p} M$ (we identify $i_{*}\left(e_{i}\right)$ with $e_{i}$ for $\left.i=1, . ., k\right)$. We have

$$
\begin{aligned}
|\tilde{S}|_{\tilde{g}}^{2} & =\Sigma_{k} \tilde{S}\left(e_{i_{1}}, . ., e_{i_{r}}\right)^{2} \\
& =\Sigma_{k} S\left(i_{*}\left(e_{i_{1}}\right), . ., i_{*}\left(e_{i_{r}}\right)\right)^{2} \\
& =\Sigma_{k} S\left(e_{i_{1}}, . ., e_{i_{r}}\right)^{2}
\end{aligned}
$$

where $\Sigma_{k}$ denotes that the sum is over all $\left(i_{1}, . ., i_{r}\right)$ with $1 \leq i_{j} \leq k, j=1, . ., r$. Hence we get

$$
|\tilde{S}|_{\tilde{g}}^{2} \leq \Sigma_{n} S\left(e_{i_{1}}, . ., e_{i_{r}}\right)^{2}
$$

where the sum is now over all $\left(i_{1}, . ., i_{r}\right)$ with $1 \leq i_{j} \leq n, j=1, \ldots, r$. But the right-hand side of the above inequality is $|S|_{g}^{2}$.

We continue with the proof of the theorem. Note that the hypothesis on $s$ and the above lemma imply that

$$
s \geq|T| \geq|\tilde{T}|_{\tilde{g}} .
$$

Combining this with the stability inequality (3), we have

$$
\int_{\Sigma} \frac{|\tilde{T}|_{\tilde{g}} \phi^{2}}{2}-\int_{\Sigma} \frac{s_{\tilde{g}} \phi^{2}}{2}+\frac{1}{2} \int_{\Sigma}|B|^{2} \phi^{2} \leq \int_{\Sigma}|\nabla \phi|^{2} .
$$

Now consider the conformal function $f$ of weight -2 on $\Sigma$ defined by

$$
f:=|\tilde{T}|+|L|^{2},
$$

where $L$ is the trace-free part of the second fundamental form $B$ and we interpret the conformal invariance of $|L|^{2}$ as in example 3) in Section 1. The associated modified scalar curvature is $\sigma(h, f)=s_{h}-f_{h}$.

Since $\Sigma$ is minimal, we have $L_{\tilde{g}}=B_{\tilde{g}}$, and we can hence rewrite (15) as

$$
-\frac{1}{2} \int_{\Sigma} \sigma(\tilde{g}, f) \phi^{2} \leq \int_{\Sigma}|\nabla \phi|^{2} .
$$

Let $\triangle$ be the Laplace operator of $\tilde{g}$, and consider the equation

$$
\triangle \phi=\frac{(n-3)}{4(n-2)} \sigma(\tilde{g}, f) \phi+\lambda \phi .
$$

If this equation has a nontrivial solution $\phi$, we claim that $\lambda \leq 0$. Suppose $\lambda>0$.

As in [7, we multiply the above equation by $\phi$, integrate and use (6) to get

$$
2 \frac{(n-2)}{(n-3)} \int_{\Sigma}|\nabla \phi|^{2}=-\frac{1}{2} \int_{\Sigma} \sigma(\tilde{g}, f) \phi^{2}-2 \lambda \frac{(n-2)}{(n-3)} \int_{\Sigma} \phi^{2}<\int_{\Sigma}|\nabla \phi|^{2},
$$

which is impossible since $\phi \not \equiv 0$. 
Now define the operator

$$
L=\triangle-\frac{(n-3)}{4(n-2)} \sigma(\tilde{g}, f),
$$

and let

$$
\lambda=\inf _{\substack{\psi \in W^{1,2} \\\|\|_{2}=1}}\langle L \psi, \psi\rangle
$$

and $u>0$ be the corresponding eigenfunction. Note that since $\sigma(\tilde{g}, f)$ is, in general, Lipschitz continuous but not smooth (at the zero locus of $|T|$ ), the best regularity we can obtain for $u$ is that $u \in C^{2, \alpha}$ for any $0<\alpha<1$. This is sufficient for our purposes. By definition, $u$ satisfies

$$
L(u)=\lambda u
$$

where $\lambda \leq 0$. Multiplying the metric of $\Sigma$ by $u^{\frac{4}{n-3}}$ and using (11), we see that the modified scalar curvature of $\Sigma$ changes to

$$
-4 \frac{(n-2)}{(n-3)} u^{-\frac{(n-3)}{4(n-2)}-1} L(u)
$$

and hence is positive unless $\lambda=0$, by (9). In this case, which is (i) of Theorem 1.1 the metric $h$ is given by $h=u^{\frac{4}{n-3}} \tilde{g}$.

If $\lambda=0$, then since $u \not \equiv 0$ satisfies (7) and hence (8), we get $u \equiv$ constant and $\sigma(\tilde{g}, f) \equiv 0$, i.e. $s_{\tilde{g}}-|\tilde{T}|_{\tilde{g}}-|B|_{\tilde{g}}^{2} \equiv 0$. This completes the proof.

Here we indicate the other conclusions one can draw: note that

$$
s_{g}-s_{\tilde{g}}+|B|_{\tilde{g}}^{2} \geq|T|_{g}-|\tilde{T}|_{\tilde{g}} \geq 0 .
$$

Going back to (3) and taking $\phi \equiv$ constant, we see that $s_{g} \equiv s_{\tilde{g}}-|B|_{\tilde{g}}^{2}$ and hence $|T|_{g} \equiv|\tilde{T}|_{\tilde{g}}$ by the above inequality.

Before proceeding with the proof of Theorem 1.2 we point out an issue involving the norm $|W|$. We look at $W$ both as an element of $\operatorname{End}\left(\Lambda^{2} T_{p}^{*} M\right)$ (for instance in Corollary 1.3) and of $\otimes{ }^{4} T_{p}^{*} M$ (in the claim below). These spaces have norms that differ by a fixed scale. Since this scale is independent of dimension, it is readily seen that, for the results that we prove, this does not pose a problem.

Proof of Theorem 1.2. The proof is very similar to the proof of Theorem 1.1. The only extra point to note is that in order to get the inequality corresponding to the second part of (4), we need to know that if $\Sigma$ is totally geodesic, then $\left|W_{\tilde{g}}\right|_{\tilde{g}} \leq\left|W_{g}\right|_{g}$. This follows from the following claim, since Lemma 2.1 implies that

$$
\left|\tilde{W}_{g}\right|_{\tilde{g}} \leq\left|W_{g}\right|_{g} .
$$

CLAIM: $\left|W_{\tilde{g}}\right| \tilde{g} \leq\left|\tilde{W}_{g}\right|_{\tilde{g}}$ with equality if and only if $W_{\tilde{g}}=\tilde{W}_{g}$.

Fix a point $p \in \Sigma$, and let $R_{\tilde{g}}$ be the Riemann curvature tensor of $(\Sigma, \tilde{g})$ at $p$, regarded as an element of $S^{2} \Lambda^{2} T_{p}^{*} \Sigma$. $R_{\tilde{g}}$ decomposes orthogonally (see, for instance, [1]) as

$$
R_{\tilde{g}}=\frac{s_{\tilde{g}}}{2(n-1)(n-2)} \tilde{g} \otimes \tilde{g}+\frac{1}{n-3} z_{\tilde{g}} \oplus \tilde{g}+W_{\tilde{g}},
$$

where $z_{\tilde{g}}=R i c_{\tilde{g}}-\frac{s_{g}}{n-1} \tilde{g}$ is the trace-free part of the Ricci tensor and $₫$ denotes the Kulkarni-Nomizu product.

We note that, if $\mathfrak{A}=\left\{h \otimes \tilde{g}: h \in S^{2} T_{p}^{*} \Sigma\right\}$, then $\left\langle W_{\tilde{g}}, u\right\rangle=0$ for any $u \in \mathfrak{A}$. 
For convenience, write (11) as

$$
R_{\tilde{g}}=S_{\tilde{g}}+W_{\tilde{g}}
$$

where $S_{\tilde{g}}$ is the sum of the first two terms on the right side of (11).

On the other hand, since $\Sigma$ is totally geodesic,

$$
R_{\tilde{g}}=\tilde{R}_{g}=\tilde{S}_{g}+\tilde{W}_{g},
$$

where $R_{g}, S_{g}$ and $W_{g}$ are the corresponding quantities for $g$. Combining (12) and (13), we get (all the norms are with respect to $\tilde{g}$ )

$$
\left|\tilde{W}_{g}\right|^{2}=\left|W_{\tilde{g}}+S_{\tilde{g}}-\tilde{S}_{g}\right|^{2}=\left|W_{\tilde{g}}\right|^{2}+\left|S_{\tilde{g}}-\tilde{S}_{g}\right|^{2} .
$$

The second equality follows from the fact that since $S_{g}=h \otimes g$ for some $h$, we have $\tilde{S}=\tilde{h} \otimes \tilde{g} \in \mathfrak{A}$.

Hence $\left|\tilde{W}_{g}\right| \geq\left|W_{\tilde{g}}\right|$.

In the case of equality, we have $S_{\tilde{g}}=\tilde{S}_{g}$. (12) and (13) then imply that $W_{\tilde{g}}=\tilde{W}_{g}$. This completes the proof of the claim.

In the $\lambda=0$ case, corresponding to the last paragraph of the proof of Theorem 1.1, we get $s_{\tilde{g}}-\left|W_{\tilde{g}}\right|_{\tilde{g}} \equiv 0$. This is case (ii) in 1.2

Proceeding as before, we also get: $s_{g}=s_{\tilde{g}}$ and $\left|W_{g}\right|_{g}=\left|W_{\tilde{g}}\right|_{\tilde{g}}$. (10) then implies that $\left|W_{\tilde{g}}\right|_{\tilde{g}}=\left|\tilde{W}_{g}\right|_{\tilde{g}}$. Hence $\tilde{S}_{g}=S_{\tilde{g}}$, by the proof of the claim.

Remark. Let us consider the simple case of a Riemannian product $(M, g)=$ $\left(\Sigma \times S^{1}, \tilde{g}+d \theta^{2}\right)$ and prove that the equality $\left|W_{\tilde{g}}\right|_{\tilde{g}}=\left|W_{g}\right|_{g}$ holds if and only if $(\Sigma, \tilde{g})$ is Einstein. To see this, we check when equality holds in both the claim and (10). Equality in the claim holds if and only if $\tilde{S}_{g}=S_{\tilde{g}}$. Hence,

$$
\frac{s_{g}}{2 n(n-1)} \tilde{g} \otimes \tilde{g}+\frac{1}{n-2} \tilde{z}_{g} \otimes \tilde{g}=\frac{s_{\tilde{g}}}{2(n-1)(n-2)} \tilde{g} \otimes \tilde{g}+\frac{1}{n-3} z_{\tilde{g}} \otimes \tilde{g} .
$$

First note that $\tilde{R} i c_{g}=R i c_{\tilde{g}}$ because $R_{g}(X, N, N, Y)=0$ for any $X, Y$ in $T_{p} \Sigma$ and $N$ normal to $\Sigma$.

Hence

$$
\tilde{z}_{g}=\tilde{R} i c_{g}-\frac{s_{g}}{n} \tilde{g}=R i c_{\tilde{g}}-\frac{s_{g}}{n-1} \tilde{g}+\frac{s_{g}}{n(n-1)} \tilde{g}=z_{\tilde{g}}+\frac{s_{\tilde{g}}}{n(n-1)} \tilde{g}
$$

by using $s_{g}=s_{\tilde{g}}$ in the last step.

Substituting the above expression for $\tilde{z}_{g}$ and again using $s_{g}=s_{\tilde{g}}$ in (15), we get

$$
\frac{1}{(n-2)(n-3)} z_{\tilde{g}} \otimes \tilde{g}=0 \text {. }
$$

This holds if and only if $z_{\tilde{g}}=0$, i.e., $\tilde{g}$ is Einstein.

Assuming that $\tilde{g}$ is Einstein, we now prove equality in (10). From the proof of Lemma 2.1], it is clear that equality holds in (10) if and only if $W_{g}(X, N, N, Y)=$ $W_{g}(X, N, Z, Y)=0$ for any $X, Y, Z \in T_{p} \Sigma$.

Since $R_{g}(X, N, N, Y)=R_{g}(X, N, Z, Y)=0$ and $R_{g}=S_{g}+W_{g}$, we just have to check that $S_{g}=0$ for these vectors. This is a routine calculation involving the Kulkarni-Nomizu product, which we skip.

Note that it follows from this remark that the product metric on $\mathbb{C} P^{2} \times S^{1}$ satisfies $s \equiv 2 \sqrt{6}|W|$.

By combining Theorem 1.2 with the Böchner formula for harmonic 2-forms on a 4-manifold, we can now prove Corollary 1.3 
Proof of Corollary 1.3 Suppose $\operatorname{dim}^{2}(\Sigma, \mathbb{R})=b_{2}(\Sigma) \neq 0$. We will show that $(\Sigma, h)$, where $h$ is the induced metric, is a self-dual Kähler surface of non-negative scalar curvature.

We first show that $\Sigma$ cannot admit a metric $h$ with $s_{h}>2 \sqrt{6}|W(h)|_{h}$. Suppose $h$ is such a metric.

By Hodge theory, $H^{2}(\Sigma, \mathbb{R}) \cong \mathfrak{H}$, where $\mathfrak{H}$ is the space of harmonic 2-forms. For four-manifolds we further have $\mathfrak{H}=\mathfrak{H}_{+} \oplus \mathfrak{H}_{-}$, where $\mathfrak{H}_{+}$and $\mathfrak{H}_{-}$are the spaces of self-dual and anti-self-dual harmonic 2-forms, respectively. Let $\omega \in \mathfrak{H}_{+}$. The Böchner formula applied to $\omega$ (see, for instance, 4]) gives

$$
\frac{1}{2} \triangle|\omega|^{2}=|\nabla \omega|^{2}-2 W^{+}(\omega, \omega)+\frac{1}{3} s|\omega|^{2},
$$

where $W=W^{+}+W^{-}$is the orthogonal decomposition of $W$ into self-dual and anti-self-dual parts.

Since $W^{+}$is a trace-free symmetric operator of the three-dimensional vector bundle $\Lambda_{+}$(the bundle of self-dual 2-forms), we have, as in [4],

$$
-2 W^{+}(\omega, \omega) \geq-\frac{2 \sqrt{6}}{3}\left|W^{+}\right||\omega|^{2} .
$$

Hence we get

$$
\frac{1}{2} \triangle|\omega|^{2} \geq|\nabla \omega|^{2}+\frac{1}{3}\left(s-2 \sqrt{6}\left|W^{+}\right|\right)|\omega|^{2} \geq|\nabla \omega|^{2}+\frac{1}{3}(s-2 \sqrt{6}|W|)|\omega|^{2} .
$$

We have a similar inequality for any harmonic anti-self-dual 2-form.

Since $b_{2} \neq 0$ by assumption, there is a non-zero harmonic 2 -form $\omega$, which we assume is self-dual without loss of generality. By integrating the above inequality on $\Sigma$ we get a contradiction, since we asumed that $s>2 \sqrt{6}|W|$.

Hence, if $b_{2} \neq 0$, by Theorem 1.2 , the induced metric $h$ on $\Sigma$ satisfies $s \equiv 2 \sqrt{6}|W|$. If $\omega$ is as above, then, by (16), $\nabla \omega=0$ and $s \equiv 2 \sqrt{6}\left|W^{+}\right|$. Hence the metric $h$ is Kähler and $W^{-}=0$. If $\omega$ is anti-self-dual, then we get $\nabla \omega=0$ and $W^{+}=0$. Hence, in either case, by reversing the orientation on $\Sigma$ if necessary, $(\Sigma, h)$ is a compact self-dual Kähler surface of non-negative scalar curvature.

\section{ACKNOWLEDGEMENTS}

I would like to thank Kazuo Akutagawa and Siddartha Gadgil for several interesting and helpful discussions.

I would also like to thank the referee for various important comments.

\section{REFERENCES}

[1] A. L. Besse, Einstein Manifolds, Berlin-Heidelberg: Springer-Verlag, 1978. MR0867684 (88f:53087)

[2] A. Derdzinski, Self-dual Kähler manifolds and Einstein manifolds of dimension four, Comp. Math., 49 (1983), 405-433. MR0707181 (84h:53060)

[3] M. Gromov and B. Lawson, Positive scalar curvature and the Dirac operator on complete Riemannian manifolds, Inst. Hautes Études Sci. Publ. Math., 58 (1983), 83-196. MR0720933 (85g:58082)

[4] M. Gursky Four-manifolds with $\delta W^{+}=0$ and Einstein constants of the sphere, Math. Ann., 318 (2000), no. 3, 417-431. MR 1800764 (2002c:53074)

[5] M. Gursky and C. LeBrun, Yamabe invariants and Spin ${ }^{c}$ structures, Geom. Funct. Anal., 8 (1998), 965-977. MR 1664788 (99k:53078) 
[6] A. Lichnerowicz, Spineurs harmoniques, C. R. Acad. Sci. Paris, 257 (1963), 7-9. MR0156292 $(27: 6218)$

[7] R. Schoen and S.T. Yau, On the structure of manifolds with positive scalar curvature, Manuscripta Math., 28 (1979), 159-183. MR0535700|(80k:53064)

Stat-Math Unit, Indian Statistical Institute, Bangalore, India

E-mail address: harish@isibang.ac.in

Current address: Department of Mathematics, Indian Institute of Science, Bangalore 560012, India 